\title{
Moments-based tight-binding calculations of local electronic structure in InAs/GaAs quantum dots for comparison to experimental measurements
}

\author{
Jun-Qiang Lu and H. T. Johnson ${ }^{\text {a) }}$ \\ Department of Mechanical and Industrial Engineering, University of Illinois, Urbana, Illinois 61801 \\ V. D. Dasika and R. S. Goldman \\ Department of Materials Science and Engineering and Department of Electrical Engineering \\ and Computer Science, University of Michigan, Ann Arbor, Michigan 48109
}

(Received 8 August 2005; accepted 13 December 2005; published online 31 January 2006)

\begin{abstract}
Local electronic properties of InAs/GaAs nanostructures are studied using a real-space moments method $s p^{3} d^{5} s^{*}$ tight-binding approach. The order $(N)$ method is unique because it allows for accurate and highly resolved determination of local density of states that accounts for local strain, disorder, and defects, without diagonalization of the full tight-binding Hamiltonian. The effects of free surfaces and strain are first investigated by considering pure, cuboidal GaAs nanostructures. The quantum confinement in an embedded InAs quantum dot is then shown directly through the local densities of states projected on different atoms in the structure. The relationship between effective energy band gap and quantum dot size is mapped onto a simple equation. Finally, the real-space study is applied to quantum dot structures observed experimentally using scanning tunneling microscopy. Atomic positions are obtained from the images and used as input into the tight-binding calculations in order to study interfacial effects on the local electronic structure of real embedded quantum dots. (C) 2006 American Institute of Physics. [DOI: 10.1063/1.2171473]
\end{abstract}

Semiconductor nanostructures have stimulated intensive research interest, owing to their unusual electrical and optical properties. ${ }^{1,2}$ The electronic properties of nanostructures, such as the energy band gap, can be tailored by their size due to quantum confinement, which provides the fundamental principle for the development of nano-optical and electronic device applications.

As the minimum feature size of a nanostructure is reduced to the length of a few monolayers, nonuniform features such as strain, defects, and impurities become more important. These heterogeneous features lead to nonuniform electronic properties. The local properties of substructures, such as embedded nanostructures, interfaces, and impurities, differ from bulk properties or even freestanding substructure properties. To study these features experimentally, reciprocal space techniques such as x-ray diffraction, for example, which is powerful for exploring the properties of uniform bulk structures, are superseded by real-space techniques, such as scanning tunneling microscopy (STM). ${ }^{3}$ Theoretically, real-space methods that can explore the local properties of nonuniform nanostructures, such as quantum dots, are also needed.

In this letter, a new real-space theoretical study is presented: electronic properties of InAs/GaAs nanostructures are considered using a moments-method tight-binding (TB) model. This computational approach is a theoretical analog to a STM probe in that it probes the local electronic structure of an individual atom (or substructures) in real space. Thus, in this letter, results of the computational approach for a real quantum dot nanostructure are compared directly to actual atom-resolved STM results for the same structure.

In this study, an $s p^{3} d^{5} s^{*}$ TB Hamiltonian by Boykin et $a l^{4,5}$ is used, in which both the diagonal and neighboring atom Hamiltonian matrix elements are environment depen-

${ }^{a)}$ Electronic mail: htj@uiuc.edu dent. This parametrization also explicitly accounts for spinorbit interaction. Calculations based on this TB parametrization accurately reproduce the band edges and band gaps of InAs, GaAs, as well as the InGaAs alloy structures from experiments. ${ }^{4,5}$ In the present work, using this TB model, the method of moments is used to reconstruct the local density of states (LDOS) projected on individual atoms in a way that accounts for nonuniform features such as surfaces, interfaces, and point defects. The local structural environment around each atom is sampled statistically by collecting moments, or products of hopping integrals connecting random walks starting and finishing on that atom. ${ }^{6-10}$ The LDOS is then deduced from these statistical moments. In this work, 1024 Chebyshev moments are used in the kernel polynomial method combined with the maximum entropy method, which has been shown to accurately reconstruct the LDOS of carbon nanotubes. Details of this method are presented by the authors elsewhere. ${ }^{6}$ Similar to an experimental STM technique, this method provides a convenient way to explore the local properties within a large structure, and thus it can be considered as a theoretical atom probe comparable to a STM. The accuracy of this theoretical probe is dependent on the type and number of moments (or random walk steps) used in the reconstruction of the LDOS.

GaAs nanostructures.-To demonstrate the method, a series of pure, cuboidal GaAs nanostructures is first considered, as shown in Figs. 1(a) and 1(b). The Ga atoms are shown as cyan and the As atoms are shown as yellow. The $x$ direction is along (110), the $y$ direction is along $(\overline{1} 10)$, and the $z$ direction is along (001). The size of the structure is characterized by $N_{x} \times N_{y} \times N_{z}$, which corresponds to the real size $N_{x} a / \sqrt{2} \times N_{y} a / \sqrt{2} \times N_{z} a$, where $a=5.6532 \AA$ is the lattice constant of GaAs. The structure is relaxed by an atomistic valence-force-field (VFF) model. ${ }^{11-13}$

In Fig. 1(c), the LDOS curves are presented for the center $\mathrm{Ga}$ atom and As atom in a $15 \times 15 \times 11 \mathrm{GaAs}$ structure, 


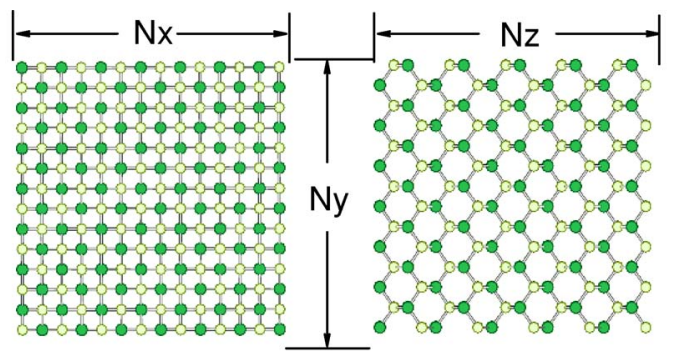

(a)

(b)
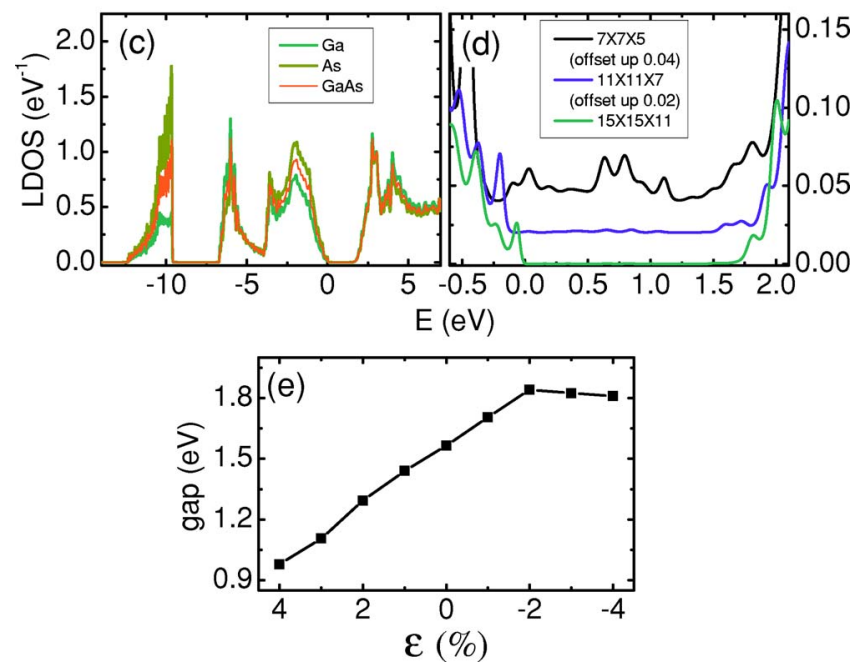

FIG. 1. (Color) (a) Top view and (b) side view of the cuboid GaAs nanostructures $N_{x} \times N_{y} \times N_{z}$. (c) The local density of states (LDOS) of the center Ga atom and As atom in GaAs $15 \times 15 \times 11$ structure. (d) The LDOS of the center Ga atoms near the Fermi energy in cuboid GaAs nanostructures with different size. (e) The band gap of the center Ga atom in GaAs $15 \times 15$ $\times 11$ structure under hydrostatic deformation $\epsilon$.

which is a freestanding cube approximately $6 \times 6 \times 6 \mathrm{~nm}$. The structure includes 9900 atoms in total. Clearly, the As atom has the largest LDOS in the valence band, while the $\mathrm{Ga}$ atom has the largest density of states (DOS) in the conduction band. The red curve in Fig. 1(c) is the average of the LDOS of the Ga and As atoms, which closely reproduces the DOS of bulk GaAs. ${ }^{14,15}$ When the size of the GaAs nanostructure is reduced, the presence of the free surface becomes important. Figure 1(d) shows the LDOS of the center Ga atoms in cuboidal GaAs nanostructures of different size. Some surface states are found among the band gap near the Fermi energy for the small structure $7 \times 7 \times 5$ (980 atoms). The surface state is associated with the unreconstructed surface structure ${ }^{14,15}$ allowed by the VFF model. As shown in Fig. 1(d), the surface states among the band gap vanish for structures larger than $15 \times 15 \times 11$. Figure $1(\mathrm{e})$ presents the band gap of the center $\mathrm{Ga}$ atom in GaAs $15 \times 15 \times 11$ structure under hydrostatic deformation, $\epsilon_{x x}=\epsilon_{y y}=\epsilon_{z z}=\epsilon$. Here, negative $\epsilon$ corresponds to hydrostatic pressure, and positive $\epsilon$ corresponds to a uniform dilation. The band gap is narrowed with a positive strain. With a negative strain, the band gap is widened first and then narrowed too, which is due to the indirect band gap. ${ }^{4}$

InAs/GaAs quantum dot structures.-As shown in Fig. 2(a), a cuboidal InAs quantum dot (QD) embedded in a cuboidal GaAs matrix is considered next. The In atoms are shown in pink. The size of the InAs QD is $D_{x} \times D_{y} \times D_{z}$. The outer boundaries of the GaAs matrix are fixed at $49 \times 49$ $\times 35$ in this study. The strain in the QD structures is relaxed (a)
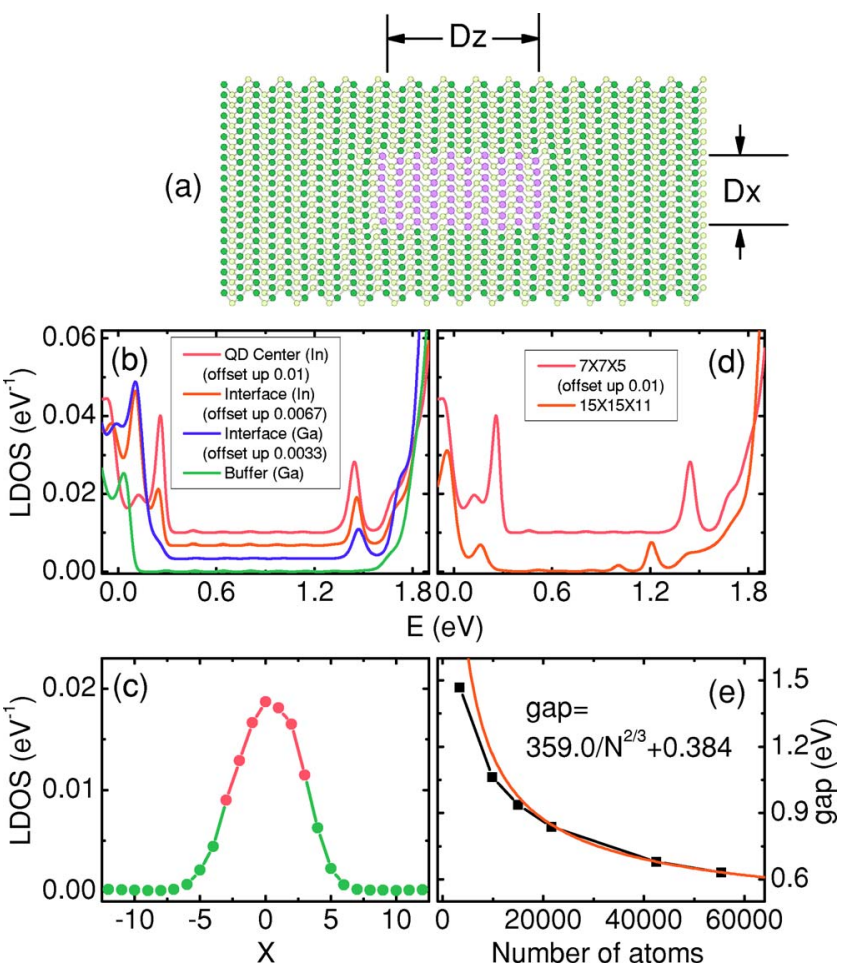

FIG. 2. (Color) (a) The center layer of the core part of relaxed structure of InAs quantum dot (QD) surrounded by GaAs buffer. (b) The LDOS of different atoms in the InAs QD $7 \times 7 \times 5$ and GaAs buffer. (c) The LDOS distribution for the energy level $1.45 \mathrm{eV}$ along the $x$ direction in the InAs QD $7 \times 7 \times 5$ and its nearby buffer. (d) The LDOS of the center In atoms of QD with different sizes. (e) The relationship between the energy gaps of InAs QD and the numbers of atoms in the QD.

using the VFF model. Figure 2(a) shows the core part of an InAs $7 \times 7 \times 5$ QD within GaAs buffers. The GaAs lattice is clearly strained due to the lattice mismatch between GaAs and InAs; the InAs quantum dot is correspondingly compressively strained.

Figure 2(b) shows the LDOS of different atoms in the InAs $7 \times 7 \times 5$ QD and GaAs buffer. Some state density is found at an energy of $1.45 \mathrm{eV}$ for the In atom in the center of the QD, and for the In and Ga atoms in the interface. Moreover, the densities decrease with increasing distance from the center of the QD. In the GaAs matrix, the LDOS at this energy level vanishes. Thus, it can be concluded that the LDOS at the energy level $1.45 \mathrm{eV}$ indicates a confined state in the InAs QD. To more plainly illustrate the confined state, the LDOS distribution for the energy level $1.45 \mathrm{eV}$ is plotted along the $x$ direction in Fig. 2(c). In this plot, the seven pink positions are In atoms and the remaining cyan positions are $\mathrm{Ga}$ atoms. The position of the center In atom in the QD is set as zero. The DOS of the confined state is largely restricted to the InAs QD region with a small tail into the nearby GaAs matrix. Mapping confined states in a quantum dot using the LDOS is a unique capability of the moments-method TB approach.

Next, size effects in InAs QDs are considered. Figure 2(d) presents the LDOS at the center In atoms of two QDs with different sizes: $7 \times 7 \times 5$ and $15 \times 15 \times 11$. Increasing the size of the QD changes the energy levels of the confined states, which effectively narrows the energy gap in the QD. The energy gap of the InAs QD is determined by both its finite size and the state of strain; due to the surrounding GaAs matrix, both the finite size and the compressive strain 

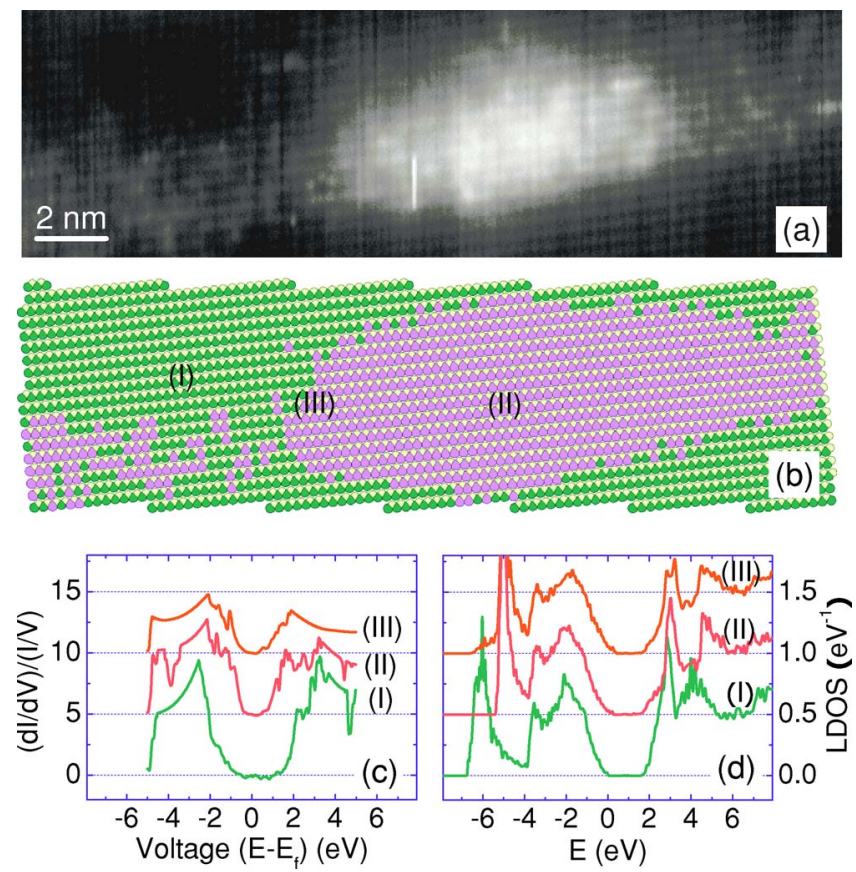

FIG. 3. (Color) (a) Atomic resolution scanning tunneling microscopy (STM) image of an InAs QD. The bright region is the QD and the dark is the GaAs buffer. (b) The atomic structure of the QD recorded from the STM image. The LDOS of (I) the Ga atom in the buffer; (II) In atom in the QD; and (III) In atom near the interface from (c) the experimental scanning tunneling spectra and (d) computational studies. Note that the curves (II) and (III) are offset up in (c) and (d).

induce a wider energy gap than in bulk InAs. In Fig. 2(e), the energy gaps of different cuboidal QDs are plotted with numbers of atoms in QDs. Clearly, the effective band gap diminishes with increasing QD size. The relationship between the effective band gap and the number of atoms in the QD can be approximately described by

$$
E_{\text {g-eff }}=359.0 / N^{2 / 3}+0.384 \text {, }
$$

where $N$ is the number of atoms in the QD. Equation (1) can be understood at a simple level by considering the InAs QD to be an ideal infinite potential well. The energy levels of the confined states in the potential well would depend on its size $L$ as $1 / L^{2}$, and thus, approximately, as $1 / N^{2 / 3}$. In the present case, when $N \rightarrow \infty$, the energy gap will be $0.384 \mathrm{eV}$, which is close to the literature value of the band gap of bulk InAs, approximately $0.37 \mathrm{eV}$. ${ }^{4,5}$ The slightly wider energy gap here is due to the compressive strain in the InAs QD as mentioned above. For the smallest QDs in Fig. 2(e), the energy gap is slightly smaller than that predicted by Eq. (1). One reason is that the InAs QD system is obviously not an ideal infinite potential well, and the confined states decay into the GaAs buffer, which lowers the energy of the confined states and leads to the smaller energy gap than that predicted by Eq. (1). For small size QD structures, this effect is important, as the tail length of the confined state in the GaAs buffer is comparable with the size of InAs QD itself. For larger QD structures, the effect of the compressive strain in the QD will be more important.

Finally, the theoretical atom probe concept is used to study an InAs QD with more complex and realistic features, as observed experimentally. Figure 3(a) shows an experimental STM image of an InAs quantum dot embedded in a GaAs matrix, where the bright region is due to higher In composition. ${ }^{16-18}$ To obtain the precise atomistic structure of the QD from the image, an algorithm based on the brightness of the STM image is used to determine the atom types and positions. After recording the atom positions from the STM image, the structure of the InAs QD and GaAs matrix is relaxed by the VFF model. The relaxed structure is shown in Fig. 3(b). Figures 3(c) and 3(d) are experimentally and computationally obtained spectra, respectively, of the matrix (mostly Ga), the QD (mostly In), and the dot/matrix interface. It is noted that the scanning tunneling spectra (STS) and the computed spectra are not from precisely the same atoms, but atoms whose environments are similar. Both the STS and computational studies show a variation in the effective band gap with position across a QD, with the smallest effective band gap near the QD center, where the In concentration is presumably highest. Additional work to compare details of the experimental and computed spectra is in progress.

In summary, a real-space computational study based on a unique moments-based TB model is demonstrated and applied to InAs/GaAs nanostructures. The effects of surfaces and strain in cuboidal GaAs nanostructures are studied. The confined states in InAs QD are plotted in real space directly by mapping the LDOS across the QD structure, and the relationship between the band gap and QD size is reduced to a simple equation. Moreover, the computational results are directly compared to results from experimental STM observations.

The authors thank M. Purfield (University of Illinois) for contributing to the STM image conversion algorithm, and J. D. Song, W. J. Choi, I. K. Han, and J. I. Lee (Nano Device Research Center, Korea Institute of Science and Technology) for growth of some of the QD samples. The support of NSF NER Grant DMR 02-10131 and ARO-MURI DAAD19-011-0462 is gratefully acknowledged.

${ }^{1}$ A. P. Alivisatos, Science 271, 933 (1996).

${ }^{2}$ J. M. Heath, Acc. Chem. Res. 32, 38 (1999).

${ }^{3}$ G. Binnig, H. Rohrer, Ch. Gerber, and E. Weibel, Appl. Phys. Lett. 40, 178 (1982)

${ }^{4}$ T. B. Boykin, G. Klimeck, R. C. Bowen, and F. Oyafuso, Phys. Rev. B 66, 125207 (2002).

${ }^{5}$ F. Oyafuso, G. Klimeck, P. Allmen, T. B. Boykin, and R. C. Bowen, Phys. Status Solidi B 239, 71 (2003).

${ }^{6}$ A. D. Schuyler, G. S. Chirikjian, J. Q. Lu, and H. T. Johnson, Phys. Rev. E 71, 046701 (2005).

${ }^{7}$ R. N. Silver and H. Roder, Phys. Rev. E 56, 4882 (1997).

${ }^{8}$ A. F. Voter, J. D. Kress, and R. N. Silver, Phys. Rev. B 53, 12733 (1996).

${ }^{9}$ F. Cyrot-Lackmann, J. Phys. Chem. Solids 29, 1235 (1968).

${ }^{10}$ F. Ducastelle and F. Cyrot-Lackmann, J. Phys. Chem. Solids 31, 1295 (1970).

${ }^{11}$ P. Keating, Phys. Rev. 145, 637 (1966).

${ }^{12}$ R. M. Martin, Phys. Rev. B 1, 4005 (1970).

${ }^{13}$ C. Pryor, J. Kim, L. W. Wang, A. J. Williamson, and A. Zunger, J. Appl. Phys. 83, 2548 (1998).

${ }^{14}$ D. J. Chadi, Phys. Rev. B 18, 1800 (1978).

${ }^{15}$ J. R. Chelikowsky and M. L. Cohen, Phys. Rev. B 20, 4150 (1979).

${ }^{16}$ B. Lita, R. S. Goldman, J. D. Phillips, and P. K. Bhattacharya, Appl. Phys. Lett. 75, 2797 (1999).

${ }^{17}$ B. Shin, B. Lita, R. S. Goldman, J. D. Phillips, and P. K. Bhattacharya, Appl. Phys. Lett. 81, 1423 (2002).

${ }^{18}$ V. D. Dasika, R. S. Goldman, J. D. Song, W. J. Choi, I. K. Han, and J. I. Lee (in preparation). 